\title{
A Spam Filtering Model of Immune Based on Multi-Agent
}

\author{
Yaping Jiang 1,a, Hao Guo 2, b ,Peigen Guo ${ }^{3, c}$
}

School of Computer and Communication Engineering, Zhengzhou University of Light Industry, Henan Zhengzhou, 450002, China

aemail:yapingjiang@163.com, bemail: guohao3456@163.com, cemail:guopeigen123@163.com

Keywords: Artificial Immune; Spam; Antigen Presentation; Multi-Agent

Abstract. According to the traditional spam filtering method effectively identify unknown characteristics and variability of the ability is not strong, according to the basic principle of biological immune system and multi agent technology proposed based on immune multi-agent spam filtering model. The model can realize the information exchange of each Agent, enhance the whole model "memory" mechanism, and effectively extract the information and variation characteristics of spam. Spam experimental simulation results show that the model and other models compared has better performance, can effectively improve the correct rate of spam model characteristics and reduce the false alarm rate.

\section{Introduction}

The information in the spam message has such features as being unable to be found and the strong ability of variation and so on. There are existing spam filtering methods, such as SVM[1], naive Bayesian 、 K-Nearest Neighborhood[2] and other text classification methods can be effective to achieve the spam detection and filtering function. But for the characteristics of variation of the mail or the emergence of new features are often unable to find and extract the characteristics of the mail, and the information is not interactive timely. In recent years, the AIS(Artificial Immune System) [3] to the biological immune system as a prototype has been successfully used, the use of artificial immune technology construction is efficient, and the anti-spam model with strong information interaction has also become the hot research.

For a single mail server receives and forwards node, it find that the new spam feature information on the entire mail system is not obvious to the entire mail system. With the increasing use of application, the network terminal load is increasing, and so is the number of spam messages. In order to solve the problem that the terminal load is large, the feature can't be extracted or delayed too long and other issues, the multi-agent technology can be applied to the spam filtering model.In the model, the different single network forwarding nodes in the mail filtering model are regarded as different process agents. Each Agent has the ability to extract the spam feature and finish alone its task alone, we can combine all agents to form a multi-agent system, which not only can be a relatively independent detection of a single agent, but also can exchange information with each other, learn to accumulate e-mail feature information, and achieve complex environment problems.

\section{Design of Immune Multi - agent Filter Model}

The Framework of the Immune multi - agent Filter Model. Immune multi-agent is very similar to the immune cell of body's immune system, which can stimulate different environments to produce a variety of different responses. Different type of immune agent is equivalent to different antibodies against different antigens. This kind of multi-agent immunity is highly instructive to solve distributed problem in complex systems. It includes the following features: autonomy,stress, efficient rate of cognitive, strong learning and memory, defense and other immune feature [4]. After analyzing the similarity between immune cells and agents, and the similarity between nature and behavior of Artificial immune system and mail filtering model.

We have established a different function of the immune agent composed of spam filtering model.The fig. 1 shows the model of Immune multi - agent spam filtering 


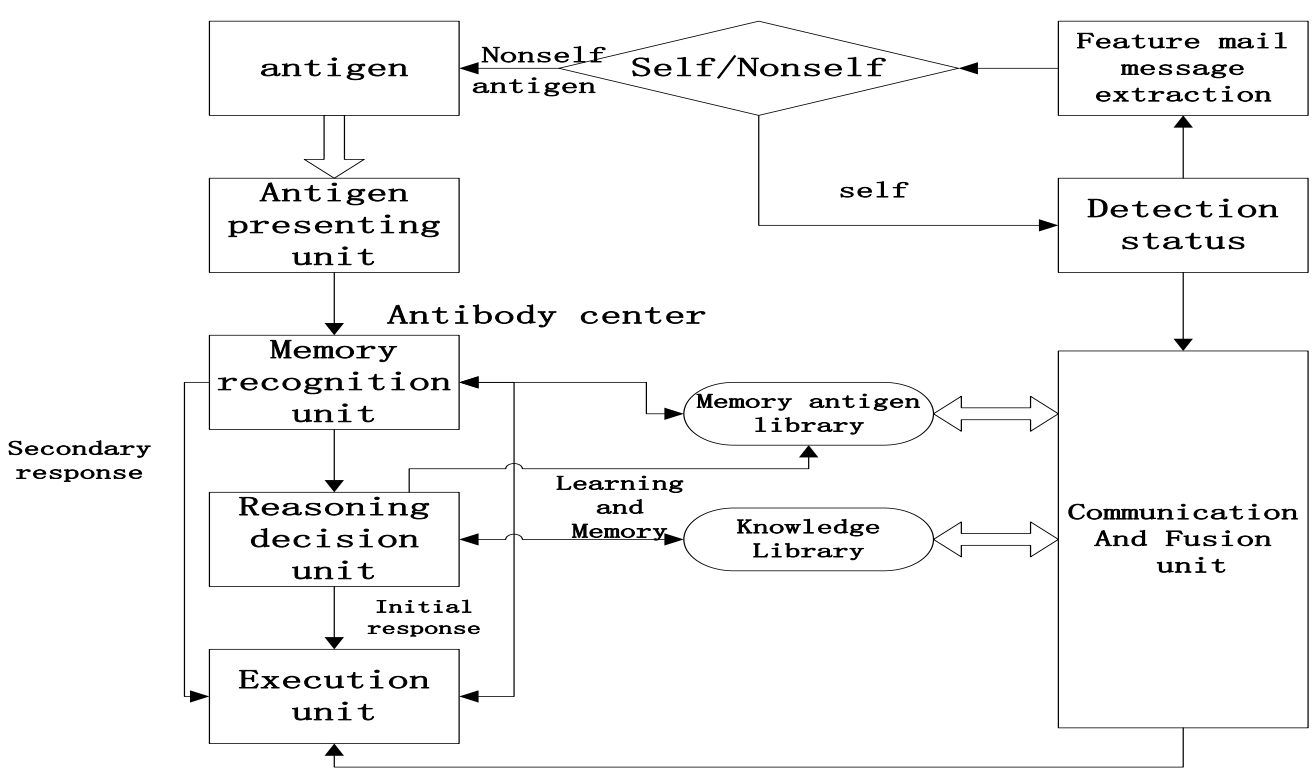

Fig.1 Immune multi-agent spam filtering model

We define the Self as the legitimate mail collection, Nonself as the spam collection. Antigen $(\mathrm{Ag})$ as a combination of Self and Nonself ( $\mathrm{Ag}=$ Self $\cup$ Nonself). The the collection of mail is $\mathrm{P}, \mathrm{p}$ is a subset of $P$, the normal mail as $H, H \in P$; and spam is $S, S \in P$, satisfy: $H \cap S=\emptyset$, $\mathrm{H} \cup \mathrm{S}=\mathrm{P}$.

In the immune agent, achieving spam filter and learning memory depends on the memory of antibody on the antigen, defense, tolerance and the mechanism of interaction between antibodies in immune system.

In the detection model, the execution layer agent, the detection layer agent, the communication layer agent and the decision layer agent are designed [5]. The immune multi-agent structure is used respectively to complete the spam model of detection, communication, implementation and decision-making tasks, which will combine different detection agents together.

Execution layer Agent: it refers to sending or receiving mail status of the Agent module in the server. It can classify mail messages and keep them in real time with the execution agent and the communication agent.

Detection layer Agent: the detection layer agent monitors the detection and communicates with the specific device among the nodes of the network server. In the model, users look for abnormal user behavior patterns, monitoring and detecting the size of the message, the number and the type, source address and destination address, the main contents of the mail and so on. If it is suspicious, it is reported to the communication agent, requesting its decision.

Communication Agent: The communication agent acts as a communication network for other Agent modules.

Decision layer Agent: it can receive module detection results from each immunoassay Agent, and determines whether the result is spam or abnormal. The module diagram is illustrated in fig. 2 . 


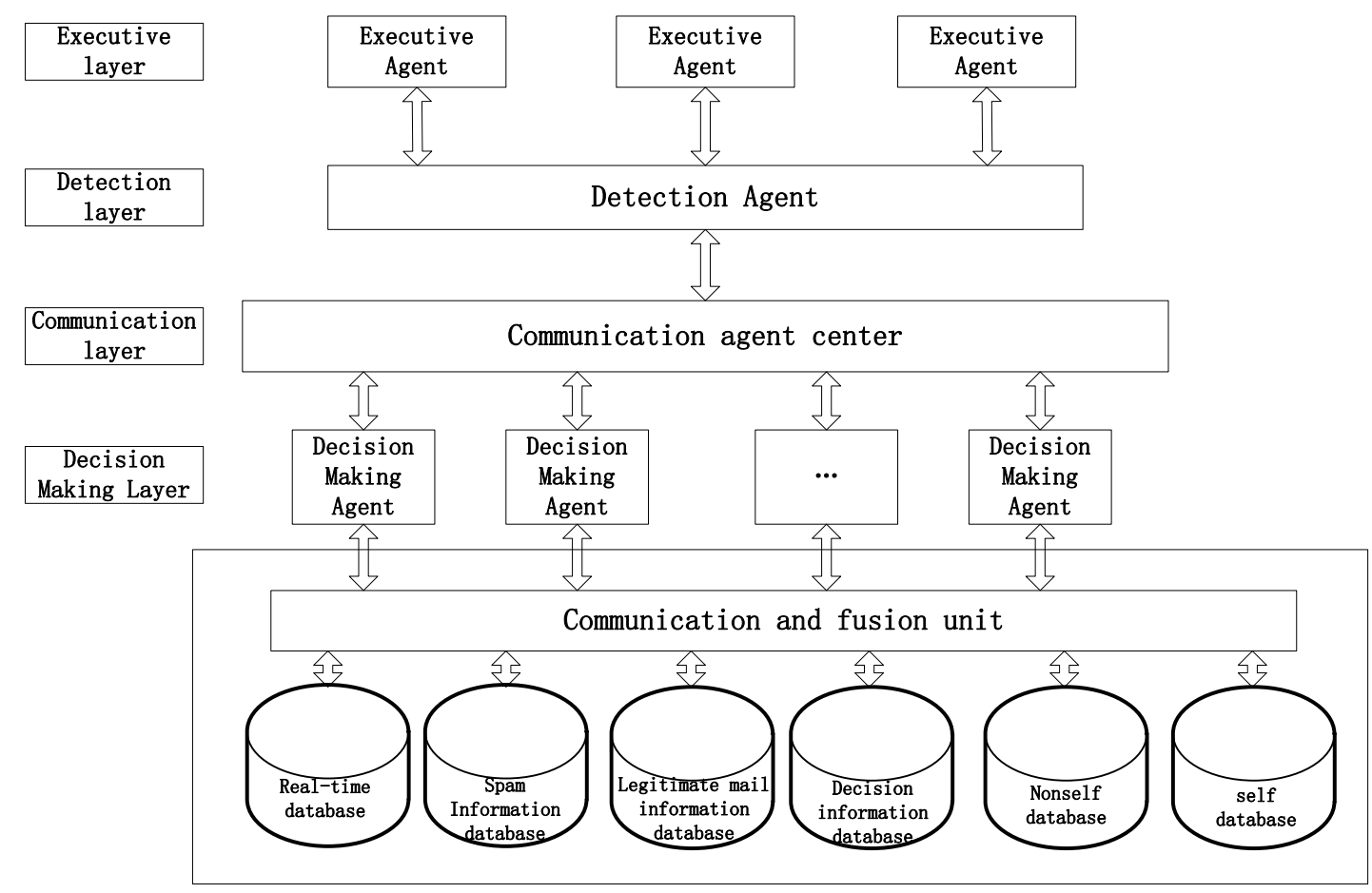

Fig.2 Immune multi-agent module

If the agent detects a suspicious spam antigen in the detection process, it is reported to the communication agent module. If the communication agent determines the antigen D containing the characteristics of the spam message, it selects several agents from the agent that is executing the agent, and shares the information contained in the D to the agent and extracts the features. When different agents receive the information instruction extracting the feature, the population that matches the antigen $\mathrm{D}$ is selected from the detector and the feature is extracted.

Implementation of Spam Filtering Based on Multi - Agent Method. To achieve spam filtering, mail data characteristics to be measured need to be transformed into computer-readable language. And then through a certain mode of filtering, it will achieve mail classification. In the mail samples, the data is submitted by antigen to form a collection of antigens, which are extracted from the specific length of the antigen presenting gene bank to obtain the characteristics of the message, that is, the antigenic determinant gene which stores in the eigenvector vector.

The antigen presenting gene is defined as the IM(Information Feature of Mail) [6] extracted from the mail subset. The length of the antigen-presenting gene set ag-l is defined as:

ag $-1=\left\{a g\left|f_{\theta}(p, i, l), l \leq i \leq\right| p\left|-l, p \in\left\{H \cup S^{\prime}\right\},\right| a g \mid=, l \in N\right\}$

Where: function $f_{e}$ performs the operation of extracting IM from the mail, 1 is the length of the extracting IM, in bits, $H^{\prime}$ ' $S^{\prime}$ ' is the subset of legitimate mail and spam,that is, extracting all lengths of land Non-repetitive IM in the mail subset, which is added into the antigen presenting gene set ag - I.Define the mail (antigen) feature set as F:

$\mathrm{F}=\left\{\left\langle x_{1}, x_{2}, x_{3, \cdots,} x_{n}\right\rangle \mid x_{i} \in\{0,1\}, p \in P, x_{i}=f_{\theta}(p, a g-l, l), i=1, \cdots, n ; n, l \in N\right\}$

Where: $x_{i}(i=1, \cdots, n)$ is the gene in the antigen presenting gene pool; ag $-l_{i}$ is the characteristic information of the extracted $\mathrm{p} \in \mathrm{P} ; \mathrm{N}$ is the dimension of the eigenvector vector, which is equal to the size of the antigen presenting gene pool; 1 is the size of the gene fragment in the antigen-presenting gene pool ag-l; The function $\mathrm{f}_{\mathrm{e}}(\mathrm{p}, \mathrm{ag}-\mathrm{l}, \mathrm{l})$ is the feature information extraction operation such as the formula (1), that is, from the $\mathrm{p} \in \mathrm{P}$ message type to extract the length of the IM string, and then calculate whether the IM appears in the corresponding antigen-presenting gene library and obtain the e-mail feature information. The function $f_{e}(p, j, l)$ indicates that the IM of length 1 is extracted from the message. With the specific antigen presenting gene library to extract the characteristics of the message, which constitutes a mail feature vector, the formula is as follows: 


$$
\begin{gathered}
\mathrm{f}_{\mathrm{e}}(p, a g-l, l)= \\
\left\{\begin{array}{l}
1, f_{\theta}(p, r, l) \in a g-l \\
0, f_{\theta}(p, r, l) \notin a g-l
\end{array}\right.
\end{gathered}
$$

After the antigen is presented, the message feature in the model memory has the same length as a computer-identifiable binary string that is as big as a gene library. Thus IT completes the process of antigen presentation. The antigen meeting the conditions presents eligible article to join the self library, or the article will add to the non-self-library, so as to ensure that the choice of eigenvector vector on the representative of the contents of the message.

\section{Simulation Experiment}

Experimental Environment and Tool. Test environment: memory 8GB, the operating system for Windows 7. In the model of mail transmission, because JAMES (Java Apache MailEnterprise Server) performance is more stable and configurable, it will select JAMES open source mail server. In the test, use the CCERT Chinese message July 2006 sample set, which contains normal mail (Ham) 9308, Spam (Spam) 20042, the numerical experiment environment for MATLAB 2014a.

Experimental Method. The data set is divided into training set and test set, 3062 e-mail (1024 normal mail and 2038 spam samples) is selected as a training set. At the beginning of the experiment, the size of the self-set is chosen to be 50, and 10 immature detectors are generated for each training. The mail filter model has 200 antigens at a time, including the ratio of Self and Nonself to 8: 2, that is, two Nonself packages are included in the 10 packets of the mail filtering model, and the initial detection samples are stored in the immune memory. And then the remaining 8,000 regular mail and 18,000 spam messages are divided into 10 groups on average to form a test set for testing.

Experimental Result. The experimental results take the average of 10 times as the final experimental data to evaluate the model. The calculated average is the efficiency of the model. The main evaluation criteria of the spam filtering model: (1) recall rate: spam detection rate;(2) the correct rate: reflect the filter model to detect the ability of spam; (3) accuracy rate: the probability that the model determines all mail correctly; (4) false rate: the probability that the model system incorrectly determines that the normal message is spam. The statistical data of the model are four indexes: correct rate, accuracy rate, recall rate and false rate, The abscissa ( $\mathrm{X}$ axis) is the number of experiments, and the ordinate is the (Y axis) percentage index value . Experimental results is shown as fig.3.

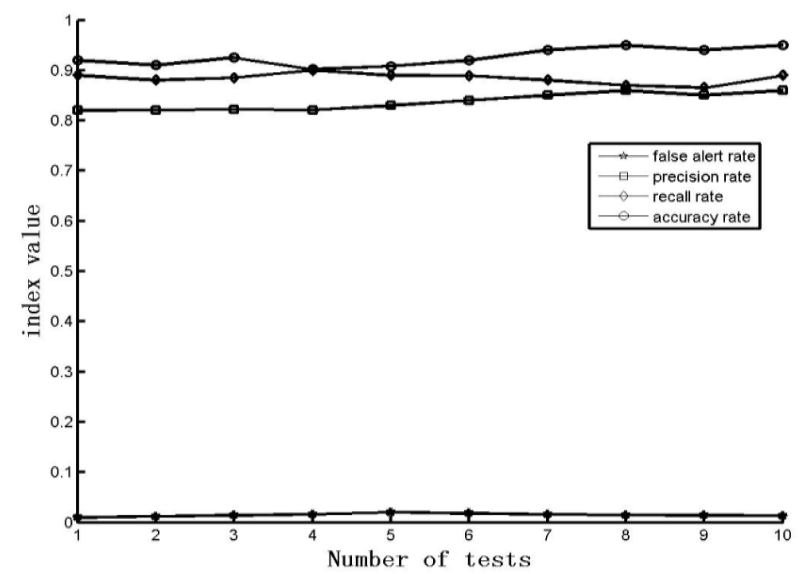

Fig.3 mail filter indicators curve

As can be seen from Figure 3, the correct rate of the model is relatively stable, the correct rate is upward trend fist, the latter part of the basic remained stable, the correct rate can reach more than $90 \%$, which Indicates that the model can be a good way to determine the spam, and the stability is higher. Precision rate in the detection process also showed an upward trend. With the antibody self-learning of the model, there is a rise in ability of model determining spam correctly and legitimate. In the recall rate, when the message appears new information features, the model has a 
better learning and memory recognition ability, which makes the ability to judge spam improve. False report rate is relatively low and stable. In this case, due to the multi-agent machine system and immune memory, information can be exchanged in time, the mail is rarely mistakenly deleted and the model has a high reliability.

\section{Conclusion}

In this paper, the spam filtering model based on immune multi-agent is given and the simulation experiment of the filtering model is realized by using MATLAB. The model realizes the information interaction of each agent and effectively extracts the information and variation characteristics of spam. The model has a high recall rate and accuracy rate, which make a certain improvement in performance. How to further enhance the efficiency of the model, will be the focus of future research.

\section{Acknowledgements}

This work was financially supported by the National Natural Science Foundation (No.61272038); Henan Science and Technology Agency-funded science and technology research projects (No.0624220084); Henan Science and Technology Department of Basic and cutting-edge technology projects (NO. 122300410255).

\section{Refereces}

[1] Clark, J.;Koprinska, I.;Poon, J. A Neural Network Based Approach to Automated E-Mail Classification[C]. Web Intelligence:Proceedings of the 2003 IEEE/WICInternational Conference on Web Intelligence. 2003:13-17.

[2] Qing J J, Mao R L, Bie R F, et al. An AIS-Based E-mail Classification Method[C]. The 2009 International Conference on Intelligent Computing, Ulsan, Korea, 2009:492-499.

[3] Li Tao. Immune - based Network Monitoring Model [J]. Journal of Computer Science, 2006, 29 (9) : 1515-1522.

[4] Liang Gang, Liu Xiao-jie, Li Tao, JIANG Yaping Yang Jin, Gong Xun. NSC: A new type of spam filter [J]. Small microcomputer system, 2008, 29 (1) :158-161.

[5] Qin Yi. Behavior - based Spam Detection Technology [J]. computer science, 2012, 39 (11): 86-89.

[6] Liu Hai-tao, Yang Jie. Research on a New Anti - spam System Based on Cloud Computing Platform[J]. Journal of Central South University（Natural Science Edition）, 2013, 44（5）: 1869-1874. 